\title{
CRISIS, DESCENTRALIZACIÓN Y GOBERNABILIDAD URBANA \\ EN EL ÁREA METROPOLITANA DE CARACAS
}

\author{
María Pilar García-Guadilla*
}

\section{INTRODUCCIÓN}

Al igual que en otros países de América Latina, la reforma política y la descentralización en Venezuela se han interpretado como procesos necesarios para hacer más eficiente el aparato administrativo del Estado; también como medios para incrementar la legitimidad política a través de la profundización de la democracia. Se supuso que la mayor eficiencia y legitimidad incidirían positivamente en los niveles de gobernabilidad.

En presencia de una descentralización parcial e incompleta, la crisis económica de fines de los ochenta hizo, que en contraste con lo esperado, el nivel de gobernabilidad general se redujera significativamente. Si bien es cierto que la descentralización a nivel local ha contribuido, en principio, a mejorar la representación y a aumentar la legitimidad del sistema político local, de no llevarse a cabo las reformas político-administrativas que requiere el Estado a nivel central, como complemento del nivel Estatal y local, se podrían reforzar las tendencias hacia la ingobernabilidad en el ámbito local-urbano.

Los efectos negativos más significativos de la crisis económico-política se han sentido en las ciudades más grandes de Venezuela, sobre todo en la ciudad capital de Caracas donde los costos socio-urbanos, producto de la configuración espacial-urbana preexistente y del modelo de desarrollo, han tendido a elevarse. Entre las causas se encuentran la implementación del proceso de descentralización de forma incompleta y parcial así como también la reducción del papel interventor del Estado sobre el espacio urbano; estos factores parecen haber estimulado el "neoliberalismo" urbano '. Por otro lado, el deterioro de la calidad de vida, el cual afecta a todos los grupos sociales, y la escasez de recursos ha suscitado conflictos entre los diferentes actores socio-urbanos, y entre éstos y el Estado. Dichos conflictos resultan difíciles de dirimir dada la baja legitimidad de los actores políticos tradicionales y la exclusión de muchos de los actores socio-urbanos del proceso de toma de decisiones.

El caso de Caracas plantea la interrogante de la gobernabilidad urbana en situaciones de aguda crisis económica, donde el Estado se desentiende del logro de los objetivos de equidad social que están implícitos en una democracia social, y donde la orientación que se privilegia en relación a la ordenación, planificación y gestión del espacio urbano es de tipo liberal.

\section{PROCESOS SOCIO-ESPACIALES Y MODELO DE DESARROLLO}

Los procesos demográficos y urbanos ocurridos en los últimos treinta y cinco años en la mayor parte de las ciudades grandes de Venezuela y, en particular, en Caracas ${ }^{2}$, se vinculan estrechamente con el modelo de desarrollo económico y político (Travieso y Urdaneta, 1971; Travieso, 1973). Dicho modelo puede definirse como un capitalismo de Estado de carácter rentista, altamente centralizado, populista, clientelar y con escasa participación de la sociedad civil (Rey, 1987; García-Guadilla 1991).

Este modelo, el cual fue el resultado de la bonanza petrolera, estimuló la localización de la población y el alto crecimiento demográfico en la Región Centro-Norte-Costera de Venezuela y, en particular, en Caracas (Travieso, 1973). Antes de los años sesenta, el crecimiento de Caracas fue el producto de las fuertes migraciones internas provenientes del medio rural y de las corrientes inmigratorias de origen europeo procedentes fundamentalmente de España, Portugal e Italia. A partir de los años setenta, la inmigración de origen europeo fue sustituida en importancia por los flujos de población provenientes de Suramérica, en particular de Colombia, y de Centroamérica (Lander y Urdaneta 1975; Chen y Picouet, 1979, 1980; Pellegrino, 1986).

La accidentada topografía de Caracas trajo como consecuencia la escasez de áreas planas desarrollables incidiendo en el alto valor de cambio del suelo urbano lo que,

* Profesora en el Departamento de Planeación Urbana de la Universidad Simón Bolívar, Caracas, Venezuela.

1. Entendemos por neoliberalismo urbano, el proceso mediante el cual se utilizan los criterios del mercado, de la libre competencia y de los actores hegemónicos para la ordenación y planificación espacial-urbana en substitución de la equidad social.

2. El contexto geográfico de Caracas se circunscribirá al Área Metropolitana Interna de Caracas; por lo tanto incluirá los municipios Sucre, Baruta, El Hatillo, Chacao y Libertador. 
a su vez, contribuyó a configurar una estructura residencial segregada y desigual socialmente debido a la dotación diferenciada de la infraestructura de servicios a favor de los zonas planificadas y en detrimento de las áreas marginales (García-Guadilla, 1993). Estos factores, junto con el modelo rentista y la lógica de localización de los actores sociales hegemónicos, contribuyeron a que se incrementaran los costos del urbanismo y se agudizaran los conflictos entre los diferentes actores socio-urbanos.

En la actualidad, la configuración socio-espacial de Caracas parece responder a la lógica neoliberal, más que a la equidad social (García-Guadilla, 1994). De forma semejante a otras ciudades latinoamericanas, Caracas ha tendido durante más de cuatro décadas a seguir los principios ecológicos que bien describiera la Escuela de Ecología Humana de la Universidad de Chicago a comienzos de siglo (Burgess, 1925; Mackenzie, 1926; Park, 1936). La visión liberal implícita en los procesos urbanos de Caracas tiende a reforzar los mecanismos de libre mercado al erigir el principio de la competencia, como el principal patrón ordenador.

En contraste con otras ciudades latinoamericanas, en Caracas se observa un fuerte intervencionismo del Estado durante el período 1950-1990, el cual se expresa en la construcción de grandes obras de vialidad e infraestructura de servicios educacionales, asistenciales y recreativos; en la generación de programas masivos de vivienda de interés social, entre los cuales se destacan los superbloques que marcaron la estructura espacial y visual de Caracas; y, finalmente, en el fuerte subsidio al financiamiento de las modernas edificaciones de condominios en propiedad horizontal que fueron la expresión de una clase media emergente (Césaris y col. 1979). No obstante, estas políticas socio-espaciales que estuvieron asociadas al fuerte capitalismo de Estado de las últimas cuatro décadas no lograron ni transformar las tendencias neoliberales de la estructura socio-espacial de Caracas ni reducir significativamente los niveles de desigualdad y pobreza. Al igual que en otras ciudades latinoamericanas, la marginalización y la pobreza de amplios sectores de la población urbana fueron en aumento expresándose en la construcción de una parte importante de la ciudad de Caracas de forma "espontánea y marginalizada” (García-Guadilla, 1985).

Mientras el Estado venezolano contó con suficientes recursos provenientes de la renta petrolera, el alto nivel de centralización existente facilitó la instrumentación de políticas socio-espaciales dirigidas a reducir los conflictos "de clase" y la segregación espacial-urbana. No obstante, al agotarse estos recursos, el Estado redujo su intervención sobre el espacio urbano y los conflictos "entre clases" y entre el Estado y los diferentes actores socio-urbanos aumentaron como producto de la fuerte competencia por los escasos recursos con que contaba el Estado para satisfacer las demandas de los actores urbanos.

La aguda crisis económica, que se manifiesta a partir de los años ochenta en Venezuela, llevaron al Estado a reducir substancialmente su impacto sobre la estructura socio-espacial de Caracas al eliminar las políticas de vivienda de interés social y la dotación de servicios gratuitos y/o subsidiados. Como consecuencia, los efectos negativos de la lógica neoliberal tendieron a expresarse con mayor fuerza en la tenencia de la tierra, en la dotación de los servicios de infraestructura urbanos y en la localización de los actores hegemónicos y de las actividades urbanas. De esta manera, se reforzaron los efectos socio-espaciales negativos del modelo de desarrollo trayendo como resultado la reducción del nivel de gobernabilidad general y de la gobernabilidad urbana, en particular.

\section{COSTOS SOCIO-ECONÓMICOS Y CONFIGURACIÓN SOCIOESPACIAL}

La configuración socio-espacial y los procesos de expansión urbana de Caracas, resultantes de las limitaciones físicas del Valle de Caracas y del modelo de desarrollo económico y socio-político, se asocian con costos económicos y socio-urbanos elevados debido a que no responden a los criterios de racionalidad económica, funcionalidad urbana y equidad social.

La infraestructura vial de Caracas que se ha adaptado a las limitaciones topográficas del Valle, junto con las preferencias de la clase alta por localizaciones en zonas de baja densidad y alta calidad ambiental que, además, fueran accesibles a la red vial principal por medio del automóvil, contribuyeron a un patrón de expansión urbana de tipo horizontal o lineal ${ }^{3}$. Este patrón resulta sumamente oneroso desde el punto de vista del urbanismo puesto que tiende a incrementar los costos de inversión en infraestructura de servicios de red y sociales y potencia, además, las deseconomías urbanas. Por otro lado, el modelo político de toma de decisiones, que se caracteriza por ser altamente centralizado y centralista, produce altos niveles de ineficiencia en la administración urbana que contribuyen, a su vez, a elevar significativamente los costos económicos urbanos.

Entre los costos resultantes de la carencia de equidad social que se observa en la configuración socio-espacial de Caracas, se encuentra la falta de accesibilidad a la vivienda ${ }^{4}$ para una gran proporción de la población urbana que vive en condiciones de pobreza debido a los altos precios del mercado y en zonas de gran inestabilidad geológica (Cartaya y D’Elia, 1991; Jiménez, 1992) ${ }^{5}$.

Otro factor que afecta la inestabilidad y por ende, los riesgos de derrumbes y deslizamientos, es el aumento acelerado de la densificación de la vivienda en las áreas marginales. Debido a su topografía sumamente accidentada y

3. La clase alta tendió a localizarse y expandirse residencialmente de acuerdo con un patrón lineal, en dirección noreste hasta los años setenta y en dirección sureste en los años posteriores. El noroeste y el sureste fueron consideradas por la clase alta y media alta como las zonas que reunían los criterios de calidad ambiental deseados.

4. La liberalización de las tasa de interés, que habían permanecido congeladas por más de treinta años, hizo inaccesibles los créditos para vivienda o para proyectos de desarrollo social.

5. Más de la mitad de la población de Caracas vive en áreas marginales donde la vivienda ha sido autoconstruida sobre terrenos pertenecientes, por lo general, al Estado que tienen escaso valor de uso debido a su inestabilidad geológica, la cual se agudiza por las filtraciones de aguas negras debido a la carencia de servicios básicos en estas zonas. 
a la escasez de terrenos para invadir, las zonas marginales de Caracas están creciendo espontáneamente y con base en iniciativas individuales mediante la densificación horizontal y vertical de las viviendas autoconstruidas (Bolívar y col. 1993). El patrón actual de densificación tiende a agudizar la inestabilidad física de la vivienda y por ende, la inestabilidad geológica del suelo, aumentando el riesgo y costos sociales.

Otro de los costos sociales del modelo es la insuficiente dotación de infraestructura de servicios básicos y sociales, además de su creciente deterioro por falta de mantenimiento de la planta física; ello es debido al elevado y creciente déficit fiscal, producto de la crisis. La carencia y el deterioro de servicios básicos tales como la dotación de agua, afectan negativamente las condiciones de salubridad de toda la población urbana al contribuir a la mayor exposición de todos los grupos socio-económicos hacia las enfermedades endémicas y epidémicas. No obstante, sus efectos más directos son en la población que vive en condiciones de pobreza.

Si bien los efectos más perniciosos del modelo de desarrollo y de la crisis económica se hacen más visibles en los barrios marginales, los costos sociales de la crisis de este modelo trascienden estos ámbitos extendiéndose a las capas medias de Caracas, las cuales resultan también afectadas por el progresivo deterioro de los servicios básicos y por la falta de infraestructura social eficiente.

\section{DESCENTRALIZACIÓN LOCAL Y GOBERNABILIDAD URBANA}

A partir de la definición de gobernabilidad como la capacidad del gobierno para ser "eficiente y eficaz" (Arbós y Giner 1993); es decir, para satisfacer las demandas de la población y así lograr el apoyo y la legitimidad necesarios (García-Guadilla y Roa 1996), se puede establecer una relación entre la crisis económica y la acentuación de los problemas urbanos; también entre las dificultades del Estado venezolano para responder a las demandas y la creciente ingobernabilidad urbana (García-Guadilla y Roa, 1996,1996a).

Desde la instauración formal de la democracia en Venezuela en 1958, el sistema de partidos clientelista se constituyó en el mecanismo principal de articulación entre un Estado, paternalista y benefactor, y una sociedad poco compleja organizacionalmente cuya gobernabilidad fue asegurada a través de pactos entre las principales fuerzas sociales (De La Cruz, 1988; Gómez Calcaño y López Maya 1990). Las nuevas organizaciones sociales urbanas que desde fines de los años setenta comienzan a demandar una mayor participación en los procesos de gestión urbana, quedaron excluidas de estos pactos fundacionales de la democracia. Dentro de estas organizaciones se insertan, además de las organizaciones urbanas o asociaciones de vecinos, los grupos ecológicos, cooperativos, culturales, populares y de mujeres que por lo general se expresan en el ámbito local y reivindican la reconstrucción de un tejido político a escala humana donde el ciudadano pueda tener acceso a los bienes básicos de la ciudad a través de una efectiva participación en el diseño, gestión, implementación y evaluación de las políticas que le afectan (GarcíaGuadilla, 1994 y 1994a).

A mediados de los años ochenta, los pactos socio-políticos se debilitaron como consecuencia de la escasez de recursos por parte del Estado para garantizar el clientelismo; las medidas de ajuste estructural adoptadas en el año de 1989 para contrarrestar la crisis económica acentuaron los conflictos socio-políticos debido a los altos altos costos sociales que ocasionaron, sobre todo en la población más pobre. Paralelamente, aumentó la corrupción prevaleciente entre los partidos políticos y los grupos que formaban parte de la red clientelar y se redujo la capacidad de dirección y de gestión del Estado.

La consecuencia de los factores anteriores fue la mayor exclusión de amplios sectores de la población del proceso de toma de decisiones; esto llevó a algunos de los actores socio-urbanos a cuestionar las acciones del Estado y a exigir una mayor participación de forma de garantizar más equidad en la distribución de los costos económicos y sociales generados por la crisis. Paralelamente, el sistema económico disminuyó progresivamente su capacidad para "legitimar" al sistema político y el nivel de gobernabilidad se redujo puesto que el Estado no fue capaz de satisfacer las demandas de la población.

Estos problemas de gobernabilidad se manifiestan con mayor fuerza en Caracas, donde el colapso de los servicios de infraestructura básicos y sociales movilizan cada vez mas, no solamente a los grupos marginales, quienes tradicionalmente se habían movilizado en pro de estas reivindicaciones, sino también a la clase media (García-Guadilla, 1991; 1991a) que descubrió su potencial político movilizador. Ya no son solo las mujeres de las barriadas quienes se movilizaron por el deterioro y déficit de los servicios de agua o la recolección de basura sino también los hombres de ingresos bajos y los hombres y mujeres de los niveles medios. De esta forma, el tema de los servicios urbanos se ha convertido en uno de los principales problemas políticos de gobernabilidad urbana, el cual se expresa en movilizaciones sociales cada vez más frecuentes, heterogéneas en su composición y amplias en su articulación, que cuestionan la eficiencia y eficacia del gobierno por no ser capaz de dar respuesta a las demandas por servicios básicos y/o sociales (García-Guadilla 1991a).

Uno de los estallidos sociales más fuerte contra las medidas de ajuste estructural adoptadas por el gobierno de Carlos Andrés Pérez en 1989 fue el denominado "caracazo", acaecido en la ciudad de Caracas, en febrero de 1989. El caracazo tuvo como objetivo demandar en las calles lo que ni los partidos ni el Estado estaban en capacidad de ofrecer eficientemente: desde alimentos de la dieta básica hasta los artículos de consumo suntuario, que los medios de comunicación habían convertido en una necesidad; también se protestaba por el aumento de los alimentos y de las tarifas de transporte que eran, a su vez, consecuencia del alza del precio de la gasolina. El "caracazo" inaugura un estilo de protestas a través de las cuales se demandan los derechos de "ciudadanía"; es decir, se reclama el acceso a los bienes y 
beneficios que ofrece la ciudad. Puesto que la crisis del modelo rentista, clientelar y populista afectó los pactos instituídos entre los actores socio-políticos tradicionales (Naím y Piñango, 1984) sin incorporar al proceso de decisiones a los actores más afectados por las medidas de ajuste económico, esta forma de protesta también conlleva la demanda, por parte de los actores excluídos, de redefinir los mecanismos de articulación entre el Estado y la sociedad.

Otro factor contribuyente a la ingobernabilidad urbana es la incapacidad del Estado para asumir el rol de "mediador y regulador" de los conflictos urbanos y para garantizar la seguridad de los ciudadanos. Hasta fines de los años ochenta, el rol de mediador y regulador de los conflictos socio-políticos que el Estado venezolano ejercía a través de las políticas sociales, se vio favorecido por su posición como principal ente "productor" de la economía y como ente "reproductor" de la fuerza de trabajo ${ }^{6}$. No obstante, frente a la creciente inseguridad social de las grandes ciudades, el Estado se mostró incompetente para garantizar la protección de los ciudadanos y trasladó, "de facto", a entes privados, funciones que le son propias, como la seguridad de los ciudadanos. Como resultado, en Caracas se ha producido "la privatización" del espacio público y el "neoliberalismo urbano" (García-Guadilla 1993, 1994), los cuales contribuyen a la ingobernabilidad urbana al acentuar la exclusión de los habitantes de menores ingresos de los espacios públicos más favorecidos ambientalmente donde se ubican los grupos de ingresos medios y altos?

La Reforma Política, dentro de la cual se enmarca el proceso de descentralización político-administrativa del Estado (De la Cruz, 1992), fue iniciada a mediados de los años ochenta por la Comisión de Reforma del Estado (COPRE). Representa una respuesta a la crisis del modelo de desarrollo y a las demandas de la sociedad civil por lograr una mayor participación en los procesos de toma de decisiones. Una de sus expresiones más importantes fue la aprobación de la nueva Ley Orgánica del Régimen Municipal en 1989 (LORM) mediante la cual se le otorga a los municipios mayor autonomía política.

La municipalización, como se ha dado en llamar este proceso, aparentemente contribuyó a aumentar la legitimidad política de las instancias locales cuyos representantes fueron elegidos directamente frente al desprestigio del Gobierno de turno que durante el período de 1989-1992 debió enfrentar dos intentos de golpe de Estado. También contribuyó a una mayor representatividad del sistema político local al acercar el gobernante al gobernado mediante la elección directa de las figuras del alcalde y de los concejales. Además, el hecho que el alcalde y la mayoría de los concejales sean elegidos uninominalmente contribuye a que los representantes tomen más en cuenta las demandas de los electores urbanos que las de los partidos políticos.

Sin embargo, el proceso incompleto de descentralización a nivel global ha afectado negativamente la gobernabilidad en aquellos municipios que no pueden satisfacer las demandas de la población ${ }^{8}$ debido al antagonismo entre las demandas de los distintos actores y a la falta de recursos. Frente al escenario de creciente ingobernabilidad, el Estado venezolano, en lugar de intervenir para detener los procesos de deterioro de la calidad de vida y así mejorar los niveles de gobernabilidad, ha ido trasladando progresiva y selectivamente al sector privado las funciones más rentables que desempeñaba quedándose con las menos rentables. Esto ha incrementado el déficit fiscal y la incapacidad del Estado para responder a la demandas de una población cada vez más empobrecida.

Por otro lado, la descentralización entendida como un proceso político-administrativo global, se ha implantado de forma incompleta y parcial. El Estado redujo drásticamente su papel interventor en la gestión de la ciudad al trasladar muchas de esas funciones a los municipios; sin embargo, ello no implicó la transferencia de los recursos económicos necesarios para ejercer tal función debido a la aguda crisis económica. En el caso de Caracas, los cinco municipios varían grandemente en lo que respecta al tamaño de su población, los niveles socioeconómicos de sus habitantes y su capacidad económica para enfrentar las demandas por servicios básicos y de infraestructura social de la población. El hecho que sean los municipios con mayor población y menor nivel socio-económico los que tienen la menor capacidad económica, augura mayores niveles de conflicto e ingobernabilidad.

El proceso de descentralización tampoco ha compatibilizado los marcos jurídicos nacionales con la nueva normativa legal del nivel local por lo cual se crean solapamientos y numerosos conflictos de competencia que contribuyen a la ingobernabilidad: la Ley Orgánica de Ordenación Urbanística (LOOU) formulada en 1987 parte de una visión centralista y frecuentemente se solapa y entra en conflicto con la Ley Orgánica del Régimen Municipal (LORM) de 1988, la cual fue formulada bajo el marco de la descentralización. Estas contradicciones afectan negativamente la aprobación de los planes, sea el de Ordenación Urbana (POU) o el de Desarrollo Urbano Local (PDUL), puesto que dificultan la negociación entre los distintos actores cuyos intereses deberían reflejarse en dichos planes. Ello se debe a que ni en el POOU ni en el PDUL existen mecanismos de participación que permitan la negociación entre actores urbanos con intereses divergentes (García-Guadilla y col. 1996b) .

El proceso de descentralización y concretamente la LORM de 1988 desmanteló algunas de las instancias de coordinación a nivel urbano, por ejemplo, la Oficina

6. De 1960-1990, el Estado se constituyó en el principal ente "productor" por ser el dueño de las industrias básicas y de actividades económicas relevantes principales; asimismo, fue el principal ente "reproductor" de la economía debido a su rol en el suministro casi gratuito de los servicios de infraestructura básicos y sociales, el subsidio directo a la alimentación y al transporte y la construcción de grandes conjuntos habitacionales de interés social.

7. La privatización del espacio urbano se refiere a la apropiación de espacios públicos por entes privados e incluye, tanto la instalación de barreras en vías públicas que impiden el paso a las personas que no residen en la zona delimitada como la apropiación de las aceras y vías peatonales por parte de los vendedores ambulantes. A pesar de su inconstitucionalidad, estas prácticas son muy frecuentes en Caracas.

8. Uno de los conflictos más recientes lo constituye los constantes enfrentamientos y conflictos entre el Municipio Libertador y los vendedores ambulantes acaecido en diciembre de 1996.

9. Las orientaciones liberales del Estado venezolano están agudizando la competencia y los conflictos entre actores urbanos con intereses divergentes dentro del mismo ámbito espacial, sea el municipio o la ciudad. 
Metropolitana de Planeamiento Urbano de Caracas (OMPU), sin que se creara un organismo capaz de cumplir dichas funciones; en la actualidad no existe para Caracas un organismo rector que regule y compatibilice los procesos de planificación y gestión de los diferentes municipios ${ }^{10}$. Con la eliminación de la OMPU, no existen instancias integradoras de los intereses fragmentados de los municipios, lo cual genera conflictos e ineficiencias en las decisiones sobre los servicios que tienen una escala urbana tales como la vialidad y el transporte (García-Guadilla y col., 1996b; De la Cruz, 1992; Vallmitjana, 1993).

La descentralización local tampoco significó la profundización de las reformas políticas necesarias para lograr la participación de la población en la gestión municipal. A pesar que el Reglamento $N^{\circ} 1$ de la LORM considera a las asociaciones de vecinos como representantes de la sociedad civil e interlocutoras con el gobierno municipal, dicha ley les asignó una participación meramente consultiva en la gestión local. Adicionalmente, la crisis parece haber desmovilizado a algunos de los actores socio-urbanos que en el pasado jugaron un papel protagónico en la defensa de la "democracia participativa" (García-Guadilla y Roa, 1996; 1996a; García-Guadilla, 1996c). En lo que respecta a las Asociaciones de Vecinos, se observa el desarrollo de una conciencia vecinal de carácter "individualista" cuyas prioridades son la defensa de la calidad de vida del condominio (Juntas de Condominio) o de la urbanización o el barrio (Juntas o Asociaciones de Vecinos) dejando en segundo lugar las interrelaciones de estos ámbitos locales con la trama urbana del Municipio o de la ciudad (GarcíaGuadilla y Roa, 1996; García-Guadilla 1996c). En el caso de Caracas, esta tendencia se extiende al gobierno local de los municipios los cuales carecen de una imagen objetivo metropolitana, o de una visión de “ciudadanía”, que les permita compatibilizar los planes locales que se elaboren.

\section{CONSIDERACIONES FINALES}

Una de las consecuencias o impactos a mediano y largo plazo de los factores señalados en este artículo es la ingobernabilidad urbana y la desdemocratización de la ciudad. Las opciones posibles que tiene una colectividad para resolver sus problemas existen, según Jordi Borja (1992), opciones que integran y opciones que excluyen. La crisis y la falta de participación del modelo de gestión urbana tienden a acentuar la opción de exclusión por encima de la de integración y, por consiguiente, se tiende a incrementar la conflictividad social.

Puesto que la configuración socio-espacial de Caracas es la expresión de los distintos actores sociales que en el interactúan, es posible prever cambios en dicha configuración que reflejen las re-estructuración de los agentes socioeconómicos producida por la crisis. La creciente segregación urbana observada en las grandes ciudades de Venezuela es la expresión de procesos socio-políticos más amplios puesto que las decisiones sobre qué opción debe de adoptarse para resolver los problemas urbanos le corresponde al Estado, en consulta y con la participación de los ciudadanos, y no puede dejarse a la libre fuerza del mercado. El caso de Caracas ha demostrado que cuando el Estado no cumple las funciones que le competen en la regulación de los procesos urbanos, son los propios ciudadanos quienes asumen el rol tendiendo a priorizar soluciones individuales o de clase que "excluyen". Las consecuencias de ello son un mayor corporativismo, segregación urbana y conflictividad social que dificultan la gobernabilidad urbana.

Para transformar las tendencias y los procesos socioespaciales existentes se requiere de un enfoque a nivel de "ciudad" que tenga como objetivo la integración, vía la participación de los distintos actores sociales. Dicha participación debe ser no solo en la producción de bienes y servicios sino también en la construcción de una ciudad con ciudadanos. La construcción de soluciones integradoras requiere que los profesionales y los movimientos sociales trabajen en conjunto dentro de marcos de negociación democráticos que incluyan a todos los actores, es decir al estado, al mercado y a los movimientos sociales.

Otras propuestas para reforzar la democracia de los ciudadanos se encuentran en la descentralización del sistema político como condición de la democracia participativa, la incorporación y legitimación de las distintas demandas e intereses de todos los actores envueltos en la problemática urbana, la legitimación del enfoque de conflicto y negociación por parte de todos los actores, incluido el propio Estado y, finalmente, incorporar el criterio de la equidad social como principio rector de la negociación de las demandas de los distintos actores.

\section{REFERENCIAS BIBLIOGRÁFICAS}

Arbós, Xavier y Giner, SALVADOR (1993). La gobernabilidad, ciudadanía y democracia en la Encrucijada Mundial. Madrid, Siglo Veintiuno de España Editores.

Bolívar, Teolinda y col. (1993). Densificación y vivienda en los barrios caraqueños. Fac. de Arquitectura. UCV.

Bidegain, G. Gabriel (1986). Estado actual de los estudios de la población en Venezuela. ILDIS-UCAB.

BORJA, Jordi (1992). "Ciudadanía, poder local y calidad de vida”. Foro nacional e Internacional sobre Reforma Urbana y Medio Ambiente 30 de mayo al 4 de junio. Río de Janeiro, Brasil.

Burgess, Ernest W. (1925) "The Growth of the City: An Introduction to a Research Project”. Robert E Park, Ernest W. Burgess, and R.D. McKenzie. The City. University of Chicago Press.

Cartaya, Vanessa y D'Elia, Yolanda (1991). Pobreza en Venezuela: Realidad y Políticas. CESAP-CISOR. Caracas.

CÉSARIS, María Nuria; GARCÍA, María-Pilar; Lluberes, Mireya; y OTEYZA, Carolina. (1979). "Políticas del Estado y marginalidad en Venezuela”. Congreso Mundial de Arquitectura Paisajística, Salvador, Bahía, Brasil.

10. Antes de la LORM de 1988, la OMPU, junto con el Ministerio de Desarrollo Urbano, debían compatibilizar los distintos procesos urbanos en un Plan de Desarrollo Urbano que asegurara la gobernabilidad espacial y administrativa de Caracas. 
Chen, Chi-Yi y Picouet, Michel (1979). Dinámica de la Población. Edición UCAB-ORSTOM. Caracas.

Chen, Chi-Yi y Picouet, Michel. 1980. "Migración internacional en Venezuela: evolución y características socio-demográficas". Migraciones Internacionales en las Américas, 1: 41-62.

De la Cruz, Rafael. 1988. En busca de un nuevo pacto social. Alfadil/Trópicos. Universidad Central de Venezuela. Caracas.

De la Cruz, Rafael (1992). "La Estrategia de la descentralización en Venezuela”. Descentralización, gobernabilidad, democracia. Rafael de la Cruz( Coord.), Editorial Nueva Sociedad.

García-Guadilla, María Pilar (1985). Unanticipated Consequences of A Planned Industrial City: The Case of Ciudad Guayana, Venezuela. Ph. D. Dissertation. University of Chicago. Cap. 1.

García-Guadilla, María Pilar (1991). "Crisis, actores y conflictos socio-políticos en la Venezuela post-saudita" en Revista Argos N. . . 14. USB. Caracas.

GarcíA-Guadilla, María Pilar (1991a). "Crisis, Estado y sociedad civil: conflictos socio-ambientales en la Venezuela post-saudita”. María Pilar García-Guadilla (Coord.) Ambiente, Estado y Sociedad. Universidad Simón Bolívar-CENDES.

García-Guadilla, María Pilar (1993). "Crisis, actores sociales y geografía urbana: segregación urbana y castas socio-ecológicas". Población y Dinámica Espacial Urbano-Rural. C. Ferrer e Irma Guillén editores. IV Encuentro de Geógrafos de América Latina. Instituto de Geografía. ULA.

García-Guadilla, María Pilar (1994). "Configuración espacial y movimientos ciudadanos: Caracas en cuatro tiempos". Las Ciudades Hablan. Tomás Rodríguez Villasante (Coord). Editorial Nueva Sociedad.

GARCíA-GuAdilla, María Pilar y Blauert, Jutta (1994a). Retos para el desarrollo y la democracia: movimientos ambientales en América Latina y Europa. Editorial Nueva Sociedad.

GARCÍA-GUADILlA, María Pilar (1994b). "Efectividad simbólica, prácticas sociales y estrategias del movimiento ambientalista venezolano: sus impactos en la democracia” en García-Guadilla, María Pilar y Blauert, Jutta. op. cit.

García-Guadilla, María Pilar y RoA, Ernesto (1996). "Gobernabilidad, cambio político y sociedad civil: el proceso constituyente en Venezuela”, Revista Vene- zolana Economía y Ciencias Sociales. Vol. 2, $\mathrm{N}^{\circ} 2-3$ p. $85-112$.

García-Guadilla, María Pilar y RoA, Ernesto (1996a). "Democracia liberal, economía de mercado y sociedad civil: las organizaciones liberales en Venezuela". I Congreso Europeo de Latinoamericanistas. Instituto de Estudios de Iberoamérica y Portugal. Salamanca, España (en prensa)

GarcíA-Guadilla, María Pilar; RoA, Ernesto y RodríGUEZ, Juan Carlos (1996b). "Modelo de Gestión, Gobernabilidad y Participación: El Municipio Chacao". I Congreso Europeo de Latinoamericanistas. Instituto de Estudios de Iberoamérica y Portugal. Salamanca, España (en prensa).

García-GuAdilla, María Pilar (1996c). Democracia, Gobernabilidad, Ciudadania y Gestión Urbana y Local. Proyecto de Investigación en curso. Decanato de Investigaciones, Universidad Simón Bolívar.

GÓmez CALCAÑO, Luis y LÓpez MAYA, Margarita. 1990. El tejido de Penélope. La Reforma del Estado en Venezuela. Editorial CENDES. Caracas.

JIMÉNEZ, Virginia. 1992. "Landslides in the squatter settlements of Caracas". Environment and Urbanization, Vol. 4, N. ${ }^{\circ} 2$ : 80-89.

LANDER, Luis y Urdaneta, Alberto. 1975. "Estudios sobre migraciones en Venezuela". Ramiro Cardona (ed.), América Latina: distribución espacial de la población. Bogotá. Corporación Centro Regional de Población.

McKenzIE, Robert D. (1926) "The Scope of Human Ecology" Publications of The American Sociological Society, XX: 141-154.

Naím, Moisés y Piñango, Ramón (1984). El caso Venezuela: una ilusión de armonía. IESA. Caracas.

PARK, Robert (1936). "Human Ecology" The American Journal of Sociology, XLII Julio:1-15.

Pellegrino, Adela. 1987. "La inmigración latinoamericana en Venezuela”. Gabriel Bidegaín (comp.). op. cit.

REy, Juan Carlos (1987). "El futuro de la democracia en Venezuela" en Varios Autores Venezuela bacia el 2000: desafios y opciones. Editorial Nueva Sociedad. Caracas.

Travieso, Fernando. 1973. Ciudad, región y subdesarrollo. Caracas. Fondo Editorial Común.

Travieso, Fernando y Urdaneta, Alberto. 1971. "Marco de referencia al desarrollo urbano en Venezuela". Cuadernos de la Sociedad Venezolana de Planificación, 84-86.

Vallmitjana, Marta (Coord.) (1993). Nuevos Escenarios para el poder local: Caracas. Editorial Nueva Sociedad.

\section{RESUMEN}

El proceso de reforma política y descentralización venezolano fueron interpretados como elementos necesarios para profundizar la democracia en este país. Sin embargo, la crisis econónica y lo incompleto del proceso de descentralización han significado a la larga un descenso en el nivel de gobernabilidad general. En este contexto, la autora analiza el caso del área metropolitana de Caracas y explica las peculiaridades de la gobernabilidad urbana en situaciones de aguda crisis económica.

\section{ABSTRACT}

The Venezuelan process of political reform and transference of competences to the regional and local levels were considered as constituent elements to deep democracy in this country. However, the economic crisis and the unfinished devolution have meant at the long run a decline in the level of general governability. In this context, the author analyzes the metroplitan area of Caracas, trying to explain the urban governability in contexts of grave economic crisis. 